\title{
Recorrido histórico del Grupo Comunicar. Un análisis desde el área de Comunicación
}

\author{
Francisco J. Caro-González*, Rocío Cruz-Díaz**, Pedro Román-Graván*** \\ *Facultad de Comunicación. Universidad de Sevilla \\ e-mail: fjcaro@us.es | ORCID iD: https://orcid.org/0000-0002-7261-9377 \\ ** Facultad de Ciencias Sociales. Universidad Pablo de Olavide \\ e-mail: mrcrudia@upo.es | ORCID iD: https://orcid.org/0000-0003-3973-7416 \\ *** Facultad de Ciencias de la Educación. Universidad de Sevilla \\ e-mail: proman@us.es | ORCID iD: https://orcid.org/0000-0002-1646-9247
}

Recibido: 15-10-20; 2a versión: 26-01-21; Aceptado: 28-01-21; Publicado: 31-01-2022

Cómo citar este artículo/Citation: Caro-González, F.J.; Cruz-Díaz, R.; Román-Graván, P. (2022). Recorrido histórico del Grupo Comunicar. Un análisis desde el área de Comunicación. Revista Española de Documentación Científica, 45 (1), e315. https://doi. org/ 10.3989/redc.2022.1.1844

Resumen: La publicación en revistas científicas de impacto constituye el eje sobre el que pivota la sociedad científica y académica. Este estudio aborda una aproximación contextual al proceso de creación y consolidación de una revista académica, la revista Comunicar situada en los puestos más altos de los rankings internacionales en las áreas de educación, comunicación y estudios culturales. El proceso se inserta en el contexto de la historia de la investigación en comunicación en España. Se lleva a cabo una estrategia de investigación cualitativa de estudio de caso único. Las evidencias utilizadas son entrevistas en profundidad y análisis documental de la propia revista. Los resultados ponen de manifiesto la importancia de los elementos contextuales y humanos en el desarrollo de esta publicación.

Palabras clave: comunicación; historia de la investigación; revistas de impacto; estudio de caso; metodología cualitativa.

\section{Historical evolution of the Comunicar Group. An analysis from the Communication area}

Abstract: The publication in high-impact scientific journals constitutes the axis around which scientific and academic society pivots. This study addresses a contextual approach to the process of creation and consolidation of an academic journal, taking the Comunicar journal, which is ranked at the highest positions in the international rankings in the areas of education, communication and cultural studies, as a case of study. The process is inserted in the context of the history of communication research in Spain. A single case qualitative research strategy is carried out using in-depth interviews and documentary analysis of the Journal itself. The results show the relevance of contextual and human elements in the development of this publication.

Keywords: communication; history of research; impact scientific journals; case study; qualitative methodology.

Copyright: $\odot 2022$ CSIC. Este es un artículo de acceso abierto distribuido bajo los términos de la licencia de uso y distribución Creative Commons Reconocimiento 4.0 Internacional (CC BY 4.0). 


\section{INTRODUCCIÓN}

La divulgación, promoción y financiación de la ciencia, y de los científicos, dependen de su capacidad para publicar los resultados de sus investigaciones en las revistas ubicadas en los puestos más elevados de los rankings internacionales. Sin embargo, los académicos rara vez se preguntan acerca de cómo nace una revista académica y cómo puede llegar a convertirse en una revista de alto impacto. Es indudable que existen factores ligados a la calidad científica, pero ese análisis es incompleto al no considerar aspectos contextuales (social, institucional, epistemológico y de estructura interna) que ayuden a comprender el proceso de gestación y consolidación de una publicación de estas características. Algunas revistas han sido analizadas desde diferentes perspectivas (Cascón-Katchadourian y otros, 2020; Villegas y otros, 2020), pero siempre tomando como evidencia los artículos publicados en ellas. En esta investigación se analiza el proceso de creación de una revista académica que, en pocos años, y con escaso apoyo institucional ha alcanzado un lugar prominente en diferentes áreas de conocimiento.

Siguiendo la línea temporal de las fases por las que ha pasado la historia de la investigación en comunicación se explica el nacimiento y consolidación de la revista Comunicar desde la perspectiva de sus promotores y considerando la influencia del contexto.

Esta revista, que en sus dos primeros números se llamó Comunica y tuvo que cambiar de nombre por el de Comunicar debido a que dicho nombre ya estaba registrado, es editada por el Grupo Comunicar, que cuenta con más de 30 años de historia.

La revista Comunicar es hoy en día un referente en lo que a revistas científicas se refiere, no solo en el área de la comunicación sino también en las áreas de educación y educomunicación (Pérez-Rodríguez, y otros 2018), pero, en esta ocasión, nos ceñiremos a su estudio desde el punto de vista de la comunicación.

La importancia de la misma ha hecho que esté incluida en más de cien bases de datos internacionales selectivas, catorce plataformas de evaluación de revistas, siete directorios selectivos, catorce hemerotecas selectivas, veintitrés portales especializados de revistas científicas, veintiséis buscadores de literatura científica Open Access, veintitrés catálogos de bibliotecas, dieciséis redes sociales, y en los cuatrocientos cincuenta y siete catálogos de bibliotecas universitarias internacionales (https:// www. revistacomunicar.com/index.php?contenido=bases-de-datos).
A modo de resumen, referenciar que la revista Comunicar se compone de un Consejo de Editores conformado por un Editor Jefe, siete Editores Adjuntos/Asociados, Editores Temáticos por cada uno de los números que publican, cinco Coeditores Internacionales; sesenta Consejeros Técnicos, cinco Consejeros Técnicos de Redacción, ocho Consejeros de Redes Sociales y Visibilidad, además de los quinientos cincuenta y ocho miembros del Comité Científico y Consejo Internacional de Revisores.

El Consejo Editorial, el Editor Jefe y los Editores Adjuntos son profesionales e investigadores de reconocida solvencia y prestigio universitario. Comunicar cuenta con una red de más de 200 expertos conformada por un Comité Científico de investigadores internacionales (más de 17 países), así como por un Consejo de Redacción, y un Consejo de Revisores, compuestos por expertos de universidades y centros de investigación, sin vinculación institucional, ni con la revista ni con la editorial, marcando la evaluación y auditoría de la revista. Todos los miembros son ajenos al Grupo Editor. El Comité Científico asesora y evalúa la publicación, avalándola científicamente y proyectándola internacionalmente.

El Comité de Redacción emite informes, propone temáticas y evalúa manuscritos. El Consejo de Revisores implementa el sistema de revisión ciega. Finalmente, el Centro de Gestión Técnica y Comercial se encuentra en la base de este organigrama. Su función es sumamente relevante, ya que se ocupa de todas las innovaciones tecnológicas, del flujo de comunicación a través de las redes sociales, las actualizaciones en las bases de datos y de las transacciones basadas en las suscripciones de instituciones o de lectores individuales así como de los acuerdos comerciales que se establezcan (Aguaded-Gómez y Fonseca-Mora, 2012).

Asimismo, todos los trabajos editados en la revista se someten a evaluaciones previas por expertos del Consejo Editorial, Comité Científico y Consejo de Redacción, investigadores todos independientes.

Para conocer en profundidad la calidad, la visibilidad y el impacto de la revista animamos al lector/a interesado/a la consulta de las siguientes referencias: Pérez-Rodríguez, y otros (2018), Fonseca-Mora y Aguaded, (2014), y Aguaded-Gómez y Fonseca-Mora (2012).

En esta investigación nos ocuparemos de analizar el proceso seguido por un grupo de investigadores para la creación y consolidación de una revista de impacto. Emplearemos la metodología de 
investigación del estudio de caso. Los resultados obtenidos suponen una aportación de indudable valor al conocimiento sobre comunicación y documentación al proporcionar la visión de parte de los actores que lo conforman. Del mismo modo, aporta evidencias subjetivas sobre el proceso de creación de una revista, enfoque que no hemos encontrado en otras publicaciones sobre esta temática.

\section{LOS PERIODOS DE LA INVESTIGACIÓN EN COMUNICACIÓN EN ESPAÑA}

Para contextualizar la historia de la revista Comunicar es preciso conocer las diferentes etapas de la investigación de la comunicación por las que ha transcurrido el grupo fundador.

Martínez Nicolás (2009) identifica tres etapas en la evolución de la investigación en comunicación en España delimitadas en tres ámbitos: social, institucional y epistemológico. No es posible entender la evolución de la investigación en comunicación sin hacer referencia a su contexto y a una variable adicional, la estructura interna de la comunidad científica. Se trata de observar la procedencia, la formación o las adscripciones epistemológicas de los investigadores (Bourdieu, 2008).

Las etapas propuestas por Martínez Nicolás son emergencia, consolidación y desarrollo. Nos ocuparemos de las 2 últimas, ya que el Grupo Comunicar aparece en 1985, a mediados de la denominada etapa de Consolidación.

A continuación, se describen brevemente las etapas de consolidación y desarrollo y se propone una tercera que recoge el contexto más actual:

\subsection{Consolidación (1980-1995)}

Estamos ante la agitación en el campo de la comunicación. Concurren diferentes factores contextuales que facilitan la "explosión de la comunicación" en España. En este período nace el Grupo Comunicar. Acorde con la evolución política, social, económica y cultural, y el desarrollo del propio sistema comunicativo, se produce una expansión importante -tanto cualitativa como cuantitativa- de estudios (Jones y otros, 2000). Nacen y se afianzan los grandes grupos de comunicación multimedia: PRISA, Grupo Zeta, Grupo Godó, Grupo Correo, Prensa Española. El estado reconoce la importancia de la información en un sistema democrático y promulga la Ley 29/1984, de 2 de agosto, por la que se regula la concesión de ayudas a empresas periodísticas y agencias informativas con el objetivo expreso de garantizar la pluralidad informativa en España. La actividad informativa y empresarial despierta el interés de los investigadores.
Otro factor clave es la liberalización de la televisión y el aumento de cadenas autonómicas y privadas. La Ley del Tercer Canal de Televisión (1983), para la puesta en funcionamiento de los canales de televisión en seis Comunidades Autónomas, dinamiza el mercado audiovisual y genera una importante demanda de profesionales, directamente o en empresas satélites. España (1986) entra en la Unión Europea asumiendo la correspondiente adaptación de normativas nacionales a requerimientos de esta institución supranacional. En 1988 se aprueba la Ley de Televisión Privada, e irrumpen en el ecosistema mediático nuevas cadenas. La programación de los nuevos entrantes en el mercado audiovisual se caracteriza por una baja calidad con la que compiten agresivamente por la tarta publicitaria. Este nuevo contexto incrementa, aún más, la demanda de profesionales cualificados.

A partir de 1989 se abren Facultades de Comunicación por toda España con el subsiguiente incremento en la demanda de docentes e investigadores y un aumento posterior de egresados. El profesorado en las universidades proviene de dos fuentes: docentes de disciplinas cercanas a la comunicación (filología, sociología, estética, empresa) y docentes del ámbito profesional. Aquí se inicia la división entre las visiones "humboldtiana" y "napoleónica" de la formación en comunicación. Ante el déficit en la formación en el campo de la teoría social y en la metodología de investigación empírica, las aportaciones más relevantes provienen de disciplinas como la sociología, psicología, ciencia política, antropología o, como es el caso del Grupo Comunicar, la pedagogía. En este periodo proliferan las tesis doctorales (460 tesis doctorales entre 1980-1989). En los 9 años siguientes, el número se duplica, 993 (Jones y otros, 2000). Del mismo modo, aumenta el número de jornadas, congresos y la edición de libros y revistas especializadas. La investigación es liderada por investigadores de Cataluña y Madrid (suponen el $70 \%$ de los investigadores). Andalucía, la región en la que nace la revista, con un $6 \%$ es la quinta región, tras Navarra y País Vasco.

\subsection{Desarrollo (1995-2010)}

Esta etapa se caracteriza por la opulencia de los medios, la migración digital y la fragmentación, se produce la convergencia tecnológica en lo digital y las redes sociales (Marzal y Casero-Ripollés, 2018). El número de centros universitarios se dispara (de 20 a 44 en poco tiempo). En 1989 la cifra de egresados en periodismo fue de 4.084 .

La primera etapa de este periodo se caracteriza por la financiación de la investigación por parte 
de los gobiernos de las comunidades autónomas que dan lugar a investigaciones de carácter local y regional, quedando al margen la investigación de la realidad americana y europea (Jones, 1998). Surgen los primeros intentos de ordenar la investigación desde el Centro de Investigación de la Comunicación (CEDIC) con la base de datos Comcat (1987-1997). El análisis del estado de la investigación en comunicación pasa por un recorrido de autores destacados según temáticas: historia; política, economía y estructura; teoría y sociología de la comunicación; tecnología de la información; documentación e información electrónica; educación; publicidad, marketing y relaciones públicas. Se constata como en el apartado de "educación" que define como "el análisis del papel educador de los medios de comunicación" (Jones, 1998) no aparece ninguna referencia al Grupo Comunicar, ni a ninguno de sus investigadores, aunque en esa fecha la presencia del grupo a nivel nacional ya era importante. Aventuramos que esta ausencia se debe al origen del grupo, desde la educación a la comunicación, por lo que en sus inicios pasaba desapercibido para los teóricos del área.

Desde el contexto institucional se produce la reforma universitaria con la aprobación de la Ley Orgánica de Universidades (LOU) de 2001, ubicando la investigación académica en el primer plano de la carrera universitaria. En 2002 se crea la ANECA (Agencia Nacional de Evaluación de la Calidad y Acreditación) endureciendo los requisitos para la promoción profesional en el ámbito académico. La trayectoria del profesorado se hace dependiente de la publicación de sus trabajos (Fernández-Quijada y Masip-Masip, 2013). Los criterios para la acreditación y la obtención de los incentivos económicos a la investigación se hacen depender casi exclusivamente de la publicación en revistas indexadas (Marzal y Casero-Ripollés, 2018). Las editoriales anglosajonas se van imponiendo y establecen los criterios de calidad a través de la creación de listados de revistas de impacto. Los gestores universitarios centran sus esfuerzos en subir puestos en los rankings internacionales universitarios y potenciar la investigación internacional. La soberanía científica española (CSIC, ANECA) y europea se supedita a los índices de impacto sostenidos por multinacionales anglosajonas (Bustamante, 2018) que privilegian sistemáticamente el inglés y a las revistas estadounidenses, marginando a la producción europea y latina.

Los investigadores españoles se percatan de la importancia de la internacionalización y comienzan, tímidamente, a participar y elaborar proyectos internacionales e interdisciplinarios. Martínez y Saperas (2011) analizan los artículos publicados en revistas específicas de comunicación entre 1998 y 2007 , constatando que el $17,6 \%$ de los autores pertenecen a instituciones no españolas. Entre las temáticas de este periodo no hay ninguna referencia a la educomunicación eje central de la revista Comunicar. Son recurrentes temas relacionados con el periodismo y la información periodística $(49,4 \%)$; y la Comunicación audiovisual (17\%). Con respecto al tipo de investigación se pone de manifiesto la escasa formación metodológica de los investigadores del área (el $62 \%$ de los artículos de carácter empírico no especifican una técnica de investigación sistemática). En 2005 existen en España 44 universidades con estudios en comunicación, en las que se imparten 113 titulaciones (Moragas I Spá, M. 2005).

En 2007 se inicia la Gran Recesión y las consiguientes políticas de austeridad, que coincide con la aplicación del Plan Bolonia (Bustamante, 2018). Esto provoca una sensible precarización del trabajo docente ( $y$ por ende investigador). Se empobrecen las convocatorias de proyectos de I+D+i. Los profesionales se ven obligados a perseguir una carrera de "BOE" vigilantes a los cambios de orientación y regulación de las agencias de calidad. El talento investigador español emigra. El Real Decreto 1312/2007 del 5 de octubre sobre la acreditación nacional para el acceso a los cuerpos docentes universitarios, endurece los requisitos para la promoción universitaria.

En enero de 2008, el programa ACADEMIA responde al mandato de generalizar la exigencia de acreditación para el acceso y progresión de la carrera académica universitaria. La publicación de trabajos en revistas científicas y, dentro de ellas, en las mejor posicionadas en los índices de impacto internacionales es el requisito indispensable (Martínez-Nicolás y Saperas-Lapiedra, 2016). El impacto de ACADEMIA genera un crecimiento exponencial de la presencia de autores españoles en las revistas internacionales de la especialidad (Masip, 2011; De Filippo, 2013; Escribà y Cortiñas, 2013; Fernández-Quijada y otros, 2013; Martínez-Nicolás, 2008).

\subsection{Expansión}

A partir de 2010 se endurecen aún más las condiciones para promocionar y los sexenios (tramos de investigación), mérito ligado a incentivos económicos, se transforman en un elemento de división entre el profesorado, los que los tienen y los que no. Las posibilidades de obtención de sexenios y acreditación para las diferentes figuras docentes dependen, casi exclusivamente, de las publicaciones indexadas en las bases de datos ISI o SCO- 
PUS. Esta situación conlleva un incremento de la producción científica que responde a la lógica del mercado académico (Montero-Díaz y otros, 2018).

Una de las principales quejas de los investigadores es el déficit de revistas de impacto en el área de comunicación y, sobre todo, revistas que admiten artículos en español. A eso se une la dificultad para encontrar financiación para las investigaciones. El Área de Ciencias Sociales obtiene alrededor del $30 \%$ del presupuesto total dedicado a la financiación de proyectos de investigación y dentro de esa área, apenas un $1 \%$ se dedica a la investigación en comunicación (Caffarel, 2018).

La mercantilización de la investigación científica hace que diversos investigadores se planteen la validez del sistema de evaluación actual (Bustamante, 2018). Se han señalado los déficits conceptuales y teóricos habituales, el escaso rigor metodológico, la falta general de un compromiso social, incluyendo la despreocupación por la transferencia a la sociedad de los resultados. Desde el punto de vista epistemológico, se esfuerzan en delimitar el área de investigación (Caffarel-Serra y otros, 2017; Caffarel-Serra, 2018; Montero-Díaz y otros, 2018).

\section{OBJETIVOS}

Como se ha adelantado el objetivo de esta investigación es comprender el proceso de creación y consolidación de una revista de alto impacto de forma holística, teniendo en cuenta factores contextuales: institucionales, epistemológicos, sociales y de estructura interna (Caïs y otros, 2014). El caso seleccionado es el de la revista Comunicar, revista científica española especializada en las áreas de Educación, Comunicación y Estudios Culturales. Se ha elegido a esta revista por varias razones:

1. En 2019 era la única revista española e iberoamericana Q1 en JCR en Comunicación y en Educación y en Scopus CiteScore es Q1 en Estudios Culturales, Educación y Comunicación. En Scopus SJR es Q1 en «Cultural Studies» en Educación y Comunicación.

2. Se trata de un caso singular, en el sentido de que consigue ese nivel de excelencia sin estar ligada a las grandes editoriales anglosajonas o vinculada a una institución educativa concreta que le ofrezca respaldo como ocurre en la mayoría de las revistas que están indexadas (Repiso y otros, 2017)

Un segundo objetivo es contribuir a perfilar las etapas de la historia reciente en la investigación en comunicación propuesta por Martínez Nicolás (2009).

\section{METODOLOGÍA}

La estrategia de investigación más adecuada para alcanzar el objetivo es de carácter cualitativo, de forma longitudinal. Mediante el estudio de caso (Yin, 1993), se pretende analizar un fenómeno complejo y escasamente estudiado (Soriano Clemente, 2017), tratando de identificar, de forma holística, aquellos elementos contextuales y de estructura interna que influyen en el desarrollo de la investigación en ese campo. Yin (1993) indica que el estudio de caso es apropiado cuando los investigadores desean: 1) definir tópicos ampliamente; 2) cubrir las condiciones contextuales del fenómeno objeto de estudio; $y, 3$ ) emplear diferentes fuentes de evidencia empírica.

El caso seleccionado, por su trayectoria y relevancia en el área, es el de la revista Comunicar que actualmente es la revista española de comunicación mejor posicionada en los rankings internacionales.

Se utiliza una hibridación de técnicas de investigación: análisis de fuentes documentales, estudio del total de artículos publicados en la Revista y entrevistas en profundidad a socios y fundadores. Para el objetivo de este trabajo nos hemos centrado fundamentalmente en las entrevistas y el resto de las evidencias se han utilizado de forma complementaria dado el objetivo de la investigación y la limitación de espacio de una publicación académica. A continuación, se describen las fuentes de evidencia:

- Entrevistas en profundidad. Se han realizado cinco entrevistas semi-dirigidas a 5 socios del grupo, todos ellos con más de 15 años de antigüedad en el mismo. Dos de ellas se han aplicado a miembros fundadores. Se han seleccionado a estas personas por mantener un rol activo en el grupo durante más de 15 años. Las entrevistas se realizaron presencialmente durante el segundo semestre de 2017. La duración media ha sido de cincuenta minutos. Se observó como a partir de la cuarta entrevista se alcanzaba el nivel de saturación y las aportaciones de los socios eran redundantes. Las entrevistas fueron registradas digitalmente y posteriormente transcritas para su análisis. La guía de entrevista puede consultarse en el ANEXO 1. La codificación se ha hecho según los contextos epistemológicos, institucionales, sociales, de estructura interna y los periodos de la historia de la investigación en comunicación identificados por Martínez Nicolás (2009).

- Documentales. Se han consultado 172 documentos, se trata de diversas colecciones de publicaciones impresas y en línea sobre el Grupo Comu- 
nicar o generadas por el propio grupo: reuniones del grupo (desde 2004 a 2018), informes, firmas de convenios, estatutos, artículos de la revista, entrevistas publicadas a expertos y socios, etc. Fundamentalmente, los documentos analizados son de acceso directo en la página web (http://www.grupocomunicar.com) del grupo junto a documentos audiovisuales con entrevistas a diferentes miembros del grupo publicados en diferentes repositorios. No se ha realizado un análisis sistemático de estos documentos. Se han incorporado al software Atlas.ti y con la función de búsqueda se han identificado evidencias que complementan (confirmación de datos y fechas) la información obtenida durante las entrevistas. Cuando se ha extraído la información e integrado en el texto se han citado.

- Revista Comunicar. Otra fuente de datos ha sido el análisis de los artículos publicados en la revista Comunicar. Las variables analizadas han sido: número de artículos, autores, nacionalidad de los autores, número de la revista, año de publicación, si el autor es socio o no, y si lo es, de que provincia andaluza (el grupo se organiza en unidades provinciales). Desde el número 1 al último analizado ( $n^{\circ}$ 51) se han identificado 2.181 autores.

\section{RESULTADOS}

Tras la triangulación de las diferentes fuentes de evidencias se pueden determinar las fases evolutivas por las que ha pasado la revista Comunicar pudiendo solaparse y contribuir a la explicación de las establecidas por Martínez Nicolás (2009).

\subsection{Antecedentes (1983-1992)}

Los antecedentes de la revista Comunicar se sitúan en la primera mitad de los años 80. España se encuentra en los albores de la democracia y hay cierto florecimiento económico. En Andalucía se acaba de aprobar el Estatuto de Autonomía (28 de febrero de 1981). También se vislumbra la celebración de la Exposición Universal Iberoamericana de Sevilla en 1992. En la provincia de Huelva nace el diario Huelva Información (1983). Este medio publica un cuadernillo semanal denominado "Suplemento de Educación" (desde 1985), en el que se invita a escribir a un grupo de maestros y a algunos periodistas para que "la educación tuviese presencia en la sociedad" (Socio 4). De esta forma se forja un equipo de personas interesadas en la educación y los medios de comunicación.

A nivel institucional otro hecho marcó el nacimiento del grupo fundador de la revista. Se trata del Programa Prensa-Escuela, iniciativa de José María Maravall al frente del Ministerio de Educación y Cultura. El objetivo era dinamizar la lectura de periódicos en España "como una forma de generación de ciudadanía crítica en este contexto de madurez social y consolidación de la democracia" (Aularia, 2015). Este programa financió muchas de las actividades del grupo. Entre ellas las I Jornadas Andaluzas de Radio Escolar (se celebraron de forma continuada desde 1985 hasta 2001). Se puso de manifiesto la necesidad latente de estudiar estos temas "esperábamos 100 personas y vinieron 600" (Socio 5). Con el empuje ministerial, las instituciones públicas (administraciones locales, provinciales, regionales y nacionales) y privadas (entidades bancarias y medios de comunicación) se interesan por el vínculo entre medios y enseñanza. Se implica a los centros de profesores en la respuesta a la demanda formativa del profesorado. Los miembros del grupo inician una importante actividad formativa, y se embarcaron en la celebración de jornadas y simposios.

De forma paralela, en Almería, se crea el Grupo Aularia y la revista del mismo nombre. El primer número de la revista (1987) tuvo una buena acogida al responder a la necesidad de los docentes de información de cara a su promoción: "la primera etapa de Aularia ayudó a infinidad de profesores que estaban preparando sus oposiciones. (...) ofrecíamos mucha información sobre la administración" (Aularia, 2015).

En 1988, los educadores colaboradores del periódico Huelva Información, por sugerencia del director del rotativo, organizan un congreso provincial bajo el tópico "prensa-escuela". El congreso se centra en el uso de los medios para enseñar (fundamentalmente la prensa). Durante este periodo tiene lugar el I Certamen de Prensa Escolar: periódicos escolares hechos en toda Andalucía. En esta actividad se implican a todos los periódicos andaluces. Se incorporan al grupo jóvenes periodistas que ayudan a formar a los estudiantes en el uso de los medios de comunicación y en su elaboración. En este periodo, la investigación del grupo se orienta a la práctica docente. El público objetivo de sus estudios son profesores y maestros, y se enfocan en el uso de los medios como material didáctico.

En 1989 se celebran las IV Jornadas Andaluzas Prensa Radio y TV Escolares. Aquí se produce el encuentro de dos de sus referentes, Martínez-Salanova (Almería) y Aguaded Gómez (Huelva). A partir de este momento se crea el Grupo Pedagógico Andaluz Prensa y Educación.

En 1990 el grupo se expande a toda la comunidad autónoma andaluza e incorpora a periodistas de distintos medios con la celebración del II Congreso Andaluz de Prensa y Educación, continuación del celebrado en Benalmádena. Pérez Rodríguez 
destaca que "el grupo como tal comenzó su andadura más oficial cuando se constituyó con todas las sedes provinciales" (Aularia, 2018).

Los congresos y jornadas son una excelente vía para compartir experiencias e investigaciones, son esenciales para la generación del capital social, dan a conocer la actividad del grupo y ayudan a contactar con personas de toda Andalucía (y del resto del mundo) interesadas en la educomunicación. Es el caso de la investigadora Almansa cuando señala: "entré en el Grupo Comunicar tras haber participado varias veces en sus congresos, tras conocer a muchas de las personas que forman parte de este" (Aularia, 2015). Del mismo modo, los congresos sirven como elemento de unión "han sido hitos porque todo el grupo ha tenido que trabajar muchísimo y todo el mundo trabajaba, eso une mucho" (Socio 5).

Durante esta época, y ante el desarrollo de las cadenas autonómicas y privadas, el grupo empieza a ampliar su punto de mira a otros medios como la televisión, la radio, el cine y el cómic. El socio 3 comenta: "presenté una comunicación sobre el retroproyector, desde entonces el grupo Comunicar ha ido adaptándose a los diferentes recursos y los cambios en los medios de comunicación social de moda o más utilizados".

En 1992, el Simposio Andaluz ya contempla la diversidad mediática "Enseñar y aprender con prensa, radio y televisión". La asistencia a este evento es muy amplia y sirve para consolidar el carácter andaluz del grupo. En este mismo año se descentraliza la estructura organizativa y se constituyen las representaciones provinciales para dinamizar las diferentes actividades que se están realizando en el territorio andaluz.

\subsection{La revista Comunicar y el inicio del grupo (1993-2002)}

En 1993 se edita el primer número de la Revista de Educación en Medios de Comunicación, Comunicar. Su origen reside en las actas de los congresos. En el grupo se toma la decisión de publicarlas con antelación, para que los ponentes las tuvieran disponibles en el mismo congreso: "Fue uno de los grandes éxitos (estratégico y fundamental)" (Socio 3 ). Aunque la frecuencia de los congresos y jornadas era elevada, los socios se plantearon que "¿por qué era necesario esperar a los congresos para publicar?... y surgió la idea de la revista" (Socio 5).

Progresivamente, la revista se desvincula de las actas y adquiere una identidad propia: "Los primeros autores éramos los miembros del grupo. Enseguida empezamos a abrir el camino. Se difundió muchísimo en los congresos. La gente compraba todavía revistas" (Socio 4).

En la figura 1 se puede apreciar la variación de los autores en la Revista, teniendo en cuenta los artículos firmados por los socios según la zona geográfica. Se observa que como indica el socio

Figura 1. Evolución de la filiación provincial de los autores Andalucía Oriental y Occidental

\section{Procedencia de los Autores}

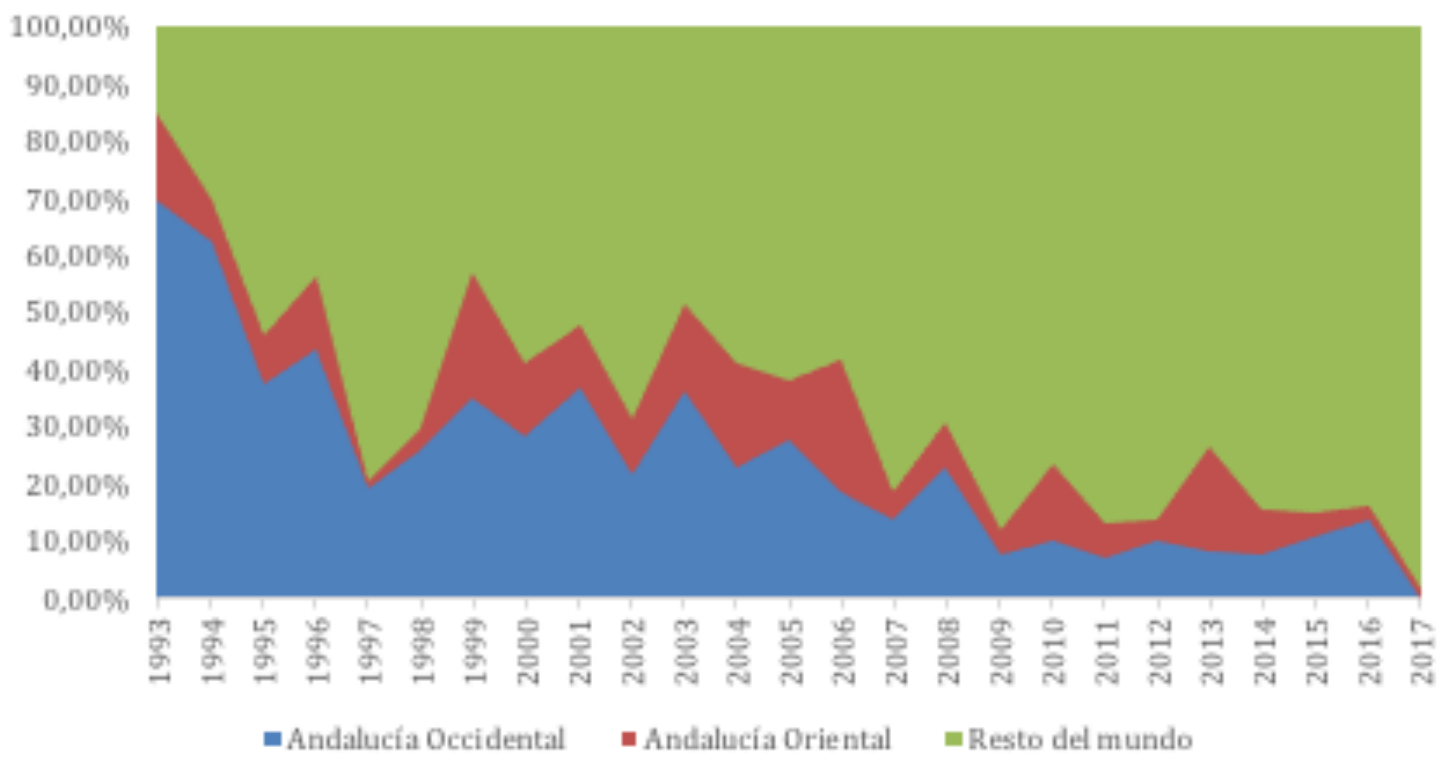


4, de un casi $90 \%$ de autorías de socios en 1993 se llega a una presencia testimonial en los volúmenes de 2017.

La venta de la revista fue la principal fuente de financiación durante ese periodo. La revista se enfocaba a investigaciones sobre la mejora docente mediante la integración de los medios de comunicación. El Consejo de Redacción originario estaba compuesto por algunos de los fundadores del grupo junto a investigadores de diversas universidades e instituciones con las que colaboran: Ministerio de Educación, Canal Sur, etc.

El grupo adquiere presencia a nivel nacional e internacional. En 1994 se celebra un congreso en Sevilla, con la presencia de más de 400 expertos de España, Francia y Portugal, bajo el título "¿Cómo enseñar y aprender la actualidad con los medios de comunicación?".

En 1996 el Grupo Pedagógico Andaluz Prensa y Educación pasa a denominarse Grupo Comunicar. Se presenta con la siguiente declaración de intenciones: "por un uso didáctico crítico, creativo y plural de los medios de comunicación en el aula y por la ética y la educación en los medios de comunicación". Se trata de "construir un proyecto integral de Educomunicación en cuatro grandes sectores: la formación, las publicaciones, la investigación y la sensibilización social" (Aularia, 2015). El logotipo del grupo, un niño que introduce su cabeza en un televisor, refleja su filosofía, la mirada crítica sobre los medios.

Se reafirma la estructura provincial con representaciones en las 8 provincias andaluzas. Se establece que no haya nunca más de 20 socios en cada provincia, "para que la gente pudiese reunirse tomando un café, la vertiente afectiva ha sido clave para mantener el grupo durante casi 30 años" (Socio 4). El contacto personal y el conocimiento entre socios, se considera la argamasa del grupo. "Siempre se ha potenciado en las reuniones provinciales y en la asamblea anual las relaciones humanas, la cercanía y el conocimiento entre los socios" (Socio 2). El socio 3 opina que: "una clave de éxito es el sentimiento de comunidad, de un proyecto común, de que las reuniones y congresos eran oportunidades para seguir unidos como colectivo de personas interesadas en hacer un poco mejor su trabajo docente".

De esta forma se promueve el trabajo colectivo y la implicación personal: "Los socios no pagaban nada, queríamos una asociación en la que la gente trabajara. No que pagaran y no trabajaran... el trabajo era el instrumento de agarre. El que no trabaja se quedaba fuera" (Socio 4). Más adelante se establecería una cuota anual destinada a hacer frente a los gastos de edición y distribución de la revista (Socio 6).

La vertiente educativa predomina en los inicios de la revista Comunicar: "los colegios e institutos son muchos y los medios de comunicación no son tantos" (Socio 2). Paulatinamente se van abordando temas relacionados con la comunicación en general. El grupo amplía el campo de estudio de la educomunicación. Se evoluciona desde la prensa en el aula al estudio de los diferentes soportes de comunicación social: la televisión, el cine, la informática, internet, las redes sociales, los videojuegos, los teléfonos móviles e inteligentes, etc.

En el n.o 17 de la revista (octubre de 2001) se modifica la maquetación para ajustarla a los formatos de imprenta, todavía estaba diseñada para las rotativas. Se inicia una segunda época, más abierta a la comunicación con mayúscula y al mundo periodístico. En 2002 se pone en marcha la ANECA y se modifican los criterios de promoción del profesorado universitario. Las publicaciones en revistas de prestigio son el principal indicador de excelencia y la revista va ganando relevancia en un área con escasas publicaciones de calidad.

\subsection{La internacionalización y la indexación de la revista Comunicar (2003- 2012)}

En el año 2003 se celebra el I Congreso Iberoamericano de Comunicación y Educación, uno de los hitos más valorados por las personas entrevistadas: "Fue un congreso con una envergadura enorme" (Socio 1) (Más de 400 comunicaciones de 20 países). Al mismo tiempo se seguía publicando la revista y cada socio trabajaba en otros proyectos personales. "Esto casi nos agotó, se trabajó muchísimo" (Socio 4) y a partir de ese momento se piensa más en la revista e "ir dejando en un segundo plano la organización de congresos" (Socio 1). Decisiones en la línea de las exigencias de calidad del trabajo científico implantadas por la comunidad universitaria.

Aunque este es el primer congreso de carácter Iberoamericano, los socios consideran que la dimensión internacional del grupo está desde sus inicios. Por ejemplo, en la revista, desde los primeros números ya destaca en su portada el carácter Iberoamericano.

Comunicar, también participa en iniciativas europeas Programa INTI 2003, como los proyectos Valores Comunes y GlocalYouth.

En el año 2005 se celebra el Congreso Hispano-Luso de Comunicación y Educación: "La televisión que queremos. Hacia una televisión de calidad". El congreso reunió a casi 700 personas y se 
editaron las comunicaciones completas en soporte digital junto a un monográfico en la revista ( $n .{ }^{\circ}$ 25). A partir de este momento el grupo se centra en la revista "todos nos ponemos a trabajar en conseguir visibilidad" (Socio 1). Detectan cómo el mundo editorial y científico está cambiando y se deciden a reducir la edición en papel.

Durante 2007 se pone en marcha el Foro "Educar la Mirada. Propuestas para Enseñar a ver Televisión", a cargo del Instituto Oficial de RTVE (Prado del Rey, instalaciones TVE). Fue el último congreso realizado. Se han organizado algunas actividades formativas (talleres), pero ya en un segundo plano. En este mismo año, la Universidad Carlos III de Madrid otorga al Grupo Comunicar el I Premio de Comunicación a la labor de Servicio Público.

También se incorpora al grupo un miembro de la spin-off EC3 de la Universidad de Granada, especializada en la evaluación de la comunicación científica para contribuir a la consolidación de la revista Comunicar como publicación de alto impacto. La revista entra en la herramienta de evaluación Journal Citation Reports (JCR), siendo la primera revista del área de comunicación en España, "nos llegó una carta impresa, casi sin esperarlo, avisando de que nos iban a incorporar. Ya en esa fecha estábamos en alta posición en el área de educación" (Socio 4).

La filosofía de la revista (y del grupo) cambia. Se prioriza la visibilidad. Se ofrece de forma gratuita y en abierto. Se apuesta por el idioma inglés para tener mayor presencia global. La revista "empezó a ser menos de la familia-grupo y más de una entidad que respondía a los cánones de una revista internacional científica" (Socio 5). Se pasó de tener casi todos los artículos de autores nacionales en los inicios de la revista a un 50\% en 2017 (Gráfico 2 ), incluso en 2018 , la proporción era del $75-80 \%$ autores internacionales.

En 2009, el n. 032 "Políticas de educación en medios", se publicó en colaboración con las Naciones Unidas y UNESCO, tanto en español como en inglés. Este mismo año, Comunicar es evaluada por primera vez en ISI y SCOPUS. Desde esta fecha ha ido mejorando su posición. En 2014 ya estaba situada en SCOPUS en el Q1 de las categorías "Communication", "Cultural Studies" y "Education".

El Socio 4 considera que "este logro no tiene tru$\mathrm{co}$, es un trabajo planificado de muchas personas. El grupo ha tenido que renunciar a muchas colecciones editoriales o a la convocatoria de congresos". Por ejemplo, la revista Aularia: "Cuando surge Comunicar decidimos dejarla en suspenso y nos pusimos a trabajar casi al completo en ella" (Socio 5). Más adelante esta revista en formato en línea se constituye como un complemento a la revista Comunicar: "Los parámetros científicos no era lo que iba buscando Aularia, sino trasladar todas esas experiencias de los docentes en el aula" (Socio 4). La filosofía de la revista es más de transferencia

Figura 2. Evolución de la filiación de los autores comparando España con el resto del mundo.

\section{País de procedencia de los autores}

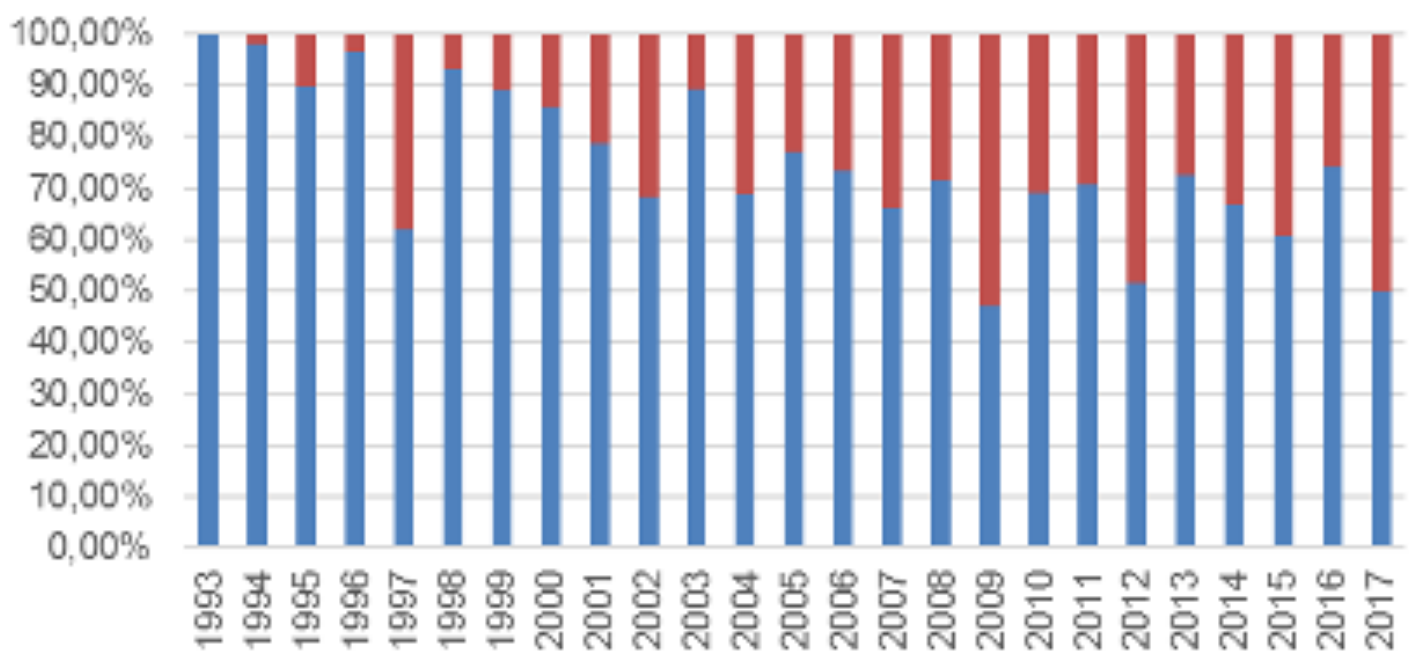

-España - Resto del Mundo 
del conocimiento: "Aularia nos permite compartir experiencias docentes sobre el uso de recursos como el cine o la música" (Socio 3 ).

La "motivación y la visión de los fundadores ha sido clave en el desarrollo del Grupo Comunicar" (Socio 2), la excelencia de la publicación se convierte en el impulso fundamental de los socios del grupo. El éxito de la revista y las exigentes normas de revisión ciega genera problemas con algunos socios e investigadores. "Todavía cuesta entenderlo a algunos catedráticos que se molestan ante un exigente proceso de revisión ciega" (Socio 4). Un ejemplo del nivel de exigencia es que en el número 47 de la revista, de 281 trabajos presentados tan solo se aceptaron 10 (tasa de rechazo del 96,44\%), participaron 147 revisores (40 internacionales y 107 nacionales) y hay autores de 6 países.

Conforme se modifican las prioridades del grupo y se transforma en una referencia global en el ámbito de la comunicación va cambiando el perfil de sus socios. Ahora son fundamentalmente profesores de universidad con formación de doctores o en vía de ello. Antes de 2008 el $57 \%$ de los socios eran doctores, desde esa fecha en adelante el porcentaje se incrementa al 76\%.

Con la idea de internacionalizar aún más el grupo, dar entrada a personas de otras zonas geográficas y "establecer alianzas estratégicas" (Socio 2) sin perder el carácter andaluz, se crea la figura del Socio de Honor de Comunicar. Desde 2010 a 2019 se han nombrado a 59 socios de 17 países diferentes.

\subsection{Cambio generacional (2013-2020)}

La revista Comunicar alcanza las mayores cotas de excelencia. Como reza en su página web:

En indexaciones activas en 2019/20, «Comunicar» es top mundial: $5^{\mathrm{a}}$ del mundo en Scopus y $9^{\mathrm{a}}$ del mundo en JCR (top $1 \%$ y $4 \%$ mundial; percentil $99 \%$ y $96 \%$ ). En JCR es Q1 ( $1^{\text {a }}$ española en Educación y $1^{a}$ en Comunicación en español). En Scopus es Q1 en Educación, Comunicación y Estudios Culturales (1 ${ }^{a}$ española); En Google Scholar Metrics es la $1^{a}$ revista indexada en español en todas las áreas. $1^{\mathrm{a}}$ en REDIB (sobre 1.038 revistas), $1^{\mathrm{a}}$ en FECYT Métricas y $1^{a}$ en Dialnet Métricas en Educación y en Comunicación.

La revista ha supuesto, en opiniones de los socios, que el grupo se convierta "en voz y árbitro de la investigación mundial sobre educomunicación" (Repiso, 2018).

El socio 5, percibe cierto riesgo en que el Grupo Comunicar acabe fagocitado por la Revista. Cons- ciente de su relevancia y de todo lo que aporta al grupo, considera que "muchas personas confunden Grupo Comunicar con la revista. La revista es uno de los instrumentos del grupo. Creo que eso es un tanto erróneo...".

Uno de los retos del futuro es abordar el cambio generacional. Actualmente la edad de los socios oscila entre los 40 y 60 años. Desde la dirección del grupo se plantea el tema del relevo. Los socios acuden atraídos por la revista y el capital social del grupo, el perfil evoluciona hacia la investigación y la obtención de resultados, estableciendo distancia sobre algunos de los principios fundacionales anteriormente citados. Algunos de los socios consideran que es necesario seguir potenciando el contacto humano, las relaciones de familiaridad, para mantener la identidad original: "Debe tener una identidad que en este momento podemos estar perdiendo, tenemos que buscar momentos de reunión más intensos, generalizar hacia otros elementos del sistema educativo" (Socio 5 ).

Por último, con relación a la internacionalización, como elemento decisivo del posicionamiento de la revista en la cima de los rankings, la revista ha empezado a lanzar sus ediciones en los idiomas que pueden incrementar su visibilidad el chino y el ruso (socio 5 ).

\section{DISCUSIÓN Y CONCLUSIONES}

En primer lugar, se destaca la validez del planteamiento de Martínez Nicolás (2009) para analizar los periodos de la evolución de la investigación en comunicación en España. Dichos periodos se presentan superpuestos a las etapas o hitos identificados en el caso estudiado (1983-2019).

A través del análisis del caso se puede apreciar la importancia de los elementos contextuales sociales, institucionales y epistemológicos en el desarrollo de la ciencia y en este caso de estudio de una revista académica.

En el caso de Comunicar se observa cómo influye el contexto social, la emergencia de nuevos medios fue clave en el desarrollo de la investigación (Caïs y otros, 2014). El diario Huelva Información tuvo un papel decisivo, también las televisiones autonómicas y privadas que despertaron la preocupación de los educadores por el impacto de su programación en la educación de la ciudadanía. Asimismo, el hecho de que se constituyese la Comunidad Autónoma de Andalucía fomentó la creación de grupos andaluces de investigación que analizaran y promocionaran la identidad regional y la divulgación de sus resultados de investigación a través de publicaciones propias. 
Por otra parte, el contexto institucional desempeña un papel clave. La creación de las Facultades de Ciencias de la Información y la llegada de los egresados provocó el aumento de investigadores en el área y del número de personas con necesidad de investigar y publicar para ingresar como profesores o mantener su estatus en la Universidad (Reig, 2014). Los congresos como estructura de divulgación permiten compartir la investigación científica durante los primeros años del grupo. Permiten darse a conocer y establecer redes de contenidos, investigadores e instituciones. Los progresivos cambios en las formas de promoción del profesorado, con la LOU y la ANECA, han provocado que actualmente tengan escasa validez curricular y se apueste por las revistas indexadas como única vía de promoción. De ahí la deriva del Grupo Comunicar hacia la revista, y el prestigio alcanzado, al lograr posicionar la revista en los puestos más altos del área de comunicación y educación.

Las decisiones políticas también tienen impacto en la evolución del Grupo Comunicar. La apuesta de Maravall por la potenciación de la lectura crítica de la prensa supuso un estímulo en el desarrollo de seminarios y acciones formativas innovadoras por todo el territorio nacional.

En el contexto epistemológico se observa como la revista tiene un enfoque inicial orientado a la investigación de la práctica educativa que se va transformando en enfoques teóricos más amplios como los estudios culturales. Las primeras investigaciones son de carácter práctico orientadas a mejorar la docencia en las aulas. Se analizan las experiencias docentes con los medios de comunicación. Poco a poco se amplían las temáticas, siempre manteniendo cierta conexión con la educomunicación. El tipo de investigación que se potencia actualmente desde la revista es de carácter empírico, con financiación competitiva y de ámbito internacional. También queda reflejado el cambio en los soportes de divulgación de la ciencia. Los manuales y monográficos pierden valor administrativo a favor de las revistas de impacto y una mayor presencia en redes sociales.

Por último, respecto a la estructura interna del grupo, puede servir como espejo de la academia del área científica en comunicación. Los rasgos que se destacan son los siguientes. En primer lugar, es necesario contar con un liderazgo que detecte las oportunidades del contexto y contribuya a posicionar el área de conocimiento y la revista en los debates de la ciencia a nivel nacional e internacional. Los responsables de los grupos de investigación tienen que reducir la incertidumbre que genera la amplitud temática del área orientando a los inves- tigadores hacia las demandas presentes y futuras de la sociedad y la ciencia, lo que se traduce en la obtención de financiación. Por otra parte, es fundamental crear una red en la que los diferentes investigadores trabajen tanto en proyectos propios como del grupo. De esta forma se amplía la red de contactos, el alcance del grupo y los conocimientos y experiencias. En el caso de Comunicar, se considera un factor aglutinador la capacidad del grupo de conectarse físicamente una vez al año y generar un sentimiento de familia entre sus socios, esto permite mantener la cohesión y realizar un esfuerzo orientado al cumplimiento de los objetivos comunes. Del mismo modo, el establecimiento de redes de investigadores es crucial en un mundo globalizado, de ahí la insistencia de los editores de la revista de mirar hacia Iberoamérica y Europa para su crecimiento (y más recientemente, Rusia y China). Los encuentros científicos ayudan a que los investigadores se conozcan en persona y se generen alianzas. La figura del Socio de Honor, o la composición del Comité Científico, el Consejo de Redacción o el Consejo Técnico sirven para estrechar esos lazos entre la comunidad científica y tejer hilos entre diferentes grupos de investigación. La principal fisura del grupo se deriva precisamente de su logro más relevante, la profesionalización de la revista que genera descontento entre algunos socios que no pueden publicar, dejan de percibirlo como un proyecto propio y optan por abandonar.

Esta investigación aporta evidencias de cómo se trasladan los enfoques teóricos sobre la evolución de la investigación en comunicación a un caso concreto. Se puede observar cómo las diferentes fases tienen capacidad explicativa de lo que ocurre con sus actores. Por otro lado, se pone de manifiesto que la ciencia es una construcción social influida en muchas ocasiones por elementos alejados de los intereses académicos. Del mismo modo se aprecia como la definición estrecha de un área de conocimiento cada vez tiene menos sentido debido a las difusas fronteras entre las áreas y la riqueza que aporta la multidisciplinariedad, esto se puede apreciar en los contenidos de la revista Comunicar y en su evaluación positiva en diferentes campos en las clasificaciones internacionales. Por último se quiere insistir en la necesidad de estudios holísticos para explicar los fenómenos sociales como es el nacimiento y consolidación de una revista de impacto, no hemos encontrado investigaciones previas con este enfoque, por lo que creemos aportamos un valor adicional y complementario a los estudios de documentación. Más allá de los datos cuantitativos es sugerente conocer las percepciones sobre las cuales los promotores han tomado las decisiones que han guiado el fenómeno de la revista Comunicar. 


\section{REFERENCIAS BIBLIOGRÁFICAS}

Aguaded-Gómez, J.I.; Fonseca-Mora, M.C. (2012). Comunicar. En: Manual de buenas prácticas en edición de revistas científicas, 11-19. Fundación Española para la Ciencia y la Tecnología, FECYT.

Aularia, Revista (2015). Temas para debate. 25 años del Grupo Comunicar: educomunicación, pasado, presente y futuro. Revista Aularia, 4(1), Enero. Almería: Ediciones Comunicar.

Aularia, Revista (2018). El Grupo Comunicar cumple treinta años de existencia eficaz. Revista Aularia, 7(1), Enero. Almería: Ediciones Comunicar.

Bourdieu, P. (2008). Homo academicus. Buenos Aires: Siglo XXI. [Edición original, Paris: Les Éditions de Minuit, 1984]

Bustamante, E. (2018). La investigación en Comunicación en España. Luces y Sombras. adComunica. Revista Científica de Estrategias, Tendencias e Innovación en Comunicación, 15, 285-288. Castellón: Asociación para el Desarrollo de la Comunicación adComunica y Universitat Jaume I. http://dx.doi.org/10.6035/21740992.2018.15.14

Caffarel-Serra, C. (2018). La metainvestigación en comunicación, una necesidad y una oportunidad. adComunica. Revista Científica de Estrategias, Tendencias e Innovación en Comunicación, 15, 293-295. Castellón: Asociación para el Desarrollo de la Comunicación adComunica y Universitat Jaume I. http://dx.doi. org/10.6035/2174-0992.2018.15.16

Caffarel-Serra, C.; Ortega-Mohedano, F.; Gaitán-Moya, J.A. (2017). Investigación en Comunicación en la universidad española en el período 2007-2014. El profesional de la información (EPI), 26(2), 218-227. https://doi.org/10.3145/epi.2017.mar.08

Caïs, J.; Folguera, L.; Formoso, C. (2014). Investigación cualitativa longitudinal. Madrid: CIS.

Cascón-Katchadourian, J.; Moral-Munoz, J. A.; Liao, H.; Cobo, M. J. (2020). Análisis bibliométrico de la $R e-$ vista Española de Documentación Científica desde su inclusión en la Web of Science (2008-2018). Revista Española de Documentación Científica, 43 (3), e267. https://doi.org/10.3989/redc.2020.3.1690

De-Filippo, D. (2013). La producción científica española en Comunicación en WOS. Las revistas indexadas en SSCI (2007-12). Comunicar. Revista Científica de Comunicación y Educación, 21(41), 25-34. Huelva. Ediciones Grupo Comunicar. https://doi.org/10.3916/ C41-2013-02.

Escribà, E.; Cortiñas, S. (2013). La internacionalización y las coautorías en las principales revistas científicas de Comunicación en España. Comunicar, Revista Científica de Comunicación y Educación, 21(41), 3544. Huelva. Ediciones Grupo Comunicar. https://doi. org/10.3916/C41-2013-03

Fernández-Quijada, D.; Masip-Masip, P. (2013). Tres décadas de investigación española en comunicación: hacia la mayoría de edad. Comunicar, Revista Científica de Comunicación y Educación, 21(41), 15-24. Huelva. Ediciones Grupo Comunicar. http://dx.doi. org/10.3916/C41-2013-01

Fonseca-Mora, M. C.; Aguaded, I. (2014). Las revistas científicas como plataformas para publicar la in- vestigación de excelencia en educación: estrategias para atracción de investigadores. RELIEVE-Revista Electrónica de Investigación y Evaluación Educativa, 20(2)

Jones, D. E. (1998). Investigación sobre comunicación en España: evolución y perspectivas. ZER-Revista de Estudios de Comunicación, 3(5).

Jones, D.E.; Baró, J.; Landa, C.; Ontalba, J.A. (2000). Investigación sobre comunicación en España. Aproximación bibliométrica a las tesis doctorales (19261998). Barcelona: ComCat.

Martínez Nicolas, M. (2008). La investigación sobre comunicación en España. Evolución histórica y retos actuales. En: Martinez-Nicolas, M. (coord.), Para investigar la comunicación. Propuestas teórico-metodológicas, 13-52. Madrid: Tecnos.

Martínez Nicolás, M. (2009). La investigación sobre comunicación en España: Evolución histórica y retos actuales. Revista Latina de Comunicación Social, 64, 1 -14. La Laguna (Tenerife): Universidad de la Laguna.

Martínez Nicolás, M.; Saperas Lapiedra, E. (2011). La investigación sobre Comunicación en España (19982007). Análisis de los artículos publicados en revistas científicas. Revista Latina de Comunicación Social, 66, 101-129. La Laguna (Tenerife): Universidad de la Laguna. http://dx.doi.org/10.4185/RLCS-66-2011926-101-129.

Martínez-Nicolás, M.; Saperas-Lapiedra, E. (2016). Objetos de estudio y orientación metodológica de la reciente investigación sobre comunicación en España (2008-2014). Análisis de los trabajos publicados en revistas científicas españolas. Revista Latina de Comunicación Social, 71, 1365-1384, La Laguna (Tenerife): Universidad de la Laguna. http://dx.doi. org/10.4185/RLCS-2016-1150es.

Martínez-Salanova, E. (2010). Historia + o - gráfica del Grupo Comunicar. Almería: Aularia- Grupo Comunicar. [https://issuu.com/grupo-comunicar/docs/historia-comunicar, consultado el 25 de julio de 2018]

Marzal Felici, J. Casero-Ripollés, A. (2018). Editorial. Investigar la comunicación: retos para el mundo académico. adComunica. Revista Científica de Estrategias, Tendencias e Innovación en Comunicación, 15, 11-17. http://dx.doi.org/10.6035/2174-0992.2018.15.1.

Masip, P. (2011). Los efectos del efecto ANECA: análisis de la producción española en comunicación en el Social Sciences Citation Index (1999-2009). En: Piñuel Raigada, J. L.; Lozano Ascencio, C. ; y García-Jiménez, A. (eds.), Investigar la comunicación en España, 649663. Madrid: Asociación Española de Investigación de la Comunicación.

Montero-Díaz, J.; Cobo, M.J.; Gutiérrez-Salcedo, M.; Segado-Boj, F.; Herrera-Viedma, E. (2018). Mapeo científico de la categoría de comunicación en WoS (19802013). Comunicar, Revista Científica de Comunicación y Educación, 55, 61-70. Huelva. Ediciones Grupo Comunicar. https://doi.org/10.3916/C55-2018-08

Moragas I Spà, M. (2005). Investigación de la comunicación y política científica en España. Ponencia presentada en la Reunión Científica de la Sociedad Española de Periodística (SEP). Santiago de Compostela, 27 y 28 de mayo de 2005 
Pérez-Rodríguez, M. A.; García-Ruiz, R.; Aguaded, I. (2018). Comunicar: calidad, visibilización e impacto/ Comunicar: Quality, Visibility and Impact. Revista Española de Pedagogía, 76(271), 481-498.

Reig, R. (2014). La investigación dependiente: crítica estructural al sistema JCR. Revista Ámbitos, 27, cuarto trimestre (invierno).

Repiso, R.; Jiménez-Contreras, E. Aguaded, I. (2017). Revistas Iberoamericanas de Educación en SciELO Citation Index y Emerging Source Citation Index. Revista Española de Documentación Científica, 40(4), e186. http://dx.doi.org/10.3989/redc.2017.4.1445
Soriano Clemente, J. (2017). Investigar la comunicación con métodos biográficos. Propuestas de estudio. Revista Historia y Comunicación Social, 22(1), 157-171. Madrid: Ediciones Complutenses.

Villegas, M. M.; Enrique-González, F.; Vincentelli, H. (2020). Cuatro Décadas de la Revista Paradigma. Producción, Autoría, Filiación Institucional y Distribución Geográfica. Paradigma. Jun2020 Anniversario: xiv-xlv. 10.37618/PARADIGMA.1011-2251.0.pXIV-XLV.id935

Yin, R.K. (1993). Applications of case study research. Applied social research methods series. London and New Delhi: Sage Publications. 


\section{ANEXO I \\ GUÍA DE ENTREVISTA}

Entrevistado:

Entrevistador:

Fecha:

Ubicación:

\section{INTRODUCCIÓN}

Exponer brevemente el objetivo de la investigación: Comprender el proceso de creación y consolidación de una revista de prestigio desde la perspectiva subjetiva de sus actores y contextualizando con el entorno institucional, político y académico.

Pedir permiso para grabar la entrevista.

\section{INDICACIONES GENERALES}

Las preguntas son orientativas. Plantear las preguntas de forma general para que sea el entrevistado el que construya su propio discurso sobre la historia del grupo y de la revista. El entrevistador debe realizar las preguntas que permitan contextualizar las respuestas en las etapas de la historia de la investigación en comunicación. El investigador debe tener visible las etapas de la historia de la investigación y los hitos previos identificados sobre el grupo para orientar la entrevista. Es particularmente interesante conocer los aspectos relacionales del grupo para contextualizar.

INICIO:

¿Cómo nace el grupo Comunicar? ¿Cuáles son sus antecedentes?

¿Quién lo conforma?

¿Han evolucionado el perfil de los socios a lo largo del tiempo?

¿Cómo se organiza el grupo? ¿Ha experimentado cambios a lo largo del tiempo?

¿Cuáles son los grandes hitos que han marcado la evolución del grupo? ¿Por qué tuvieron lugar? (El entrevistador debe tener visible la línea de tiempo con los principales hitos del grupo)

¿Qué apoyos ha recibido el grupo?

¿Cómo nace la revista Comunicar?

¿Cómo ha influido en el Grupo?

¿Por qué se apuesta por la indexación en los rankings internacionales?

¿Cómo se gestiona la revista?

¿Cuál es el futuro del grupo y de la revista?

Desea añadir algo más.

Muchas gracias por su colaboración.

NOTA:

Comprobar que se ha grabado correctamente la entrevista. En caso contrario proceder a escribir toda la información relevante que se recuerde.

En un periodo no superior a las 24 horas escribir las impresiones de la entrevista (elementos no textuales) y los elementos que más hayan llamado la atención al investigador durante la misma. 